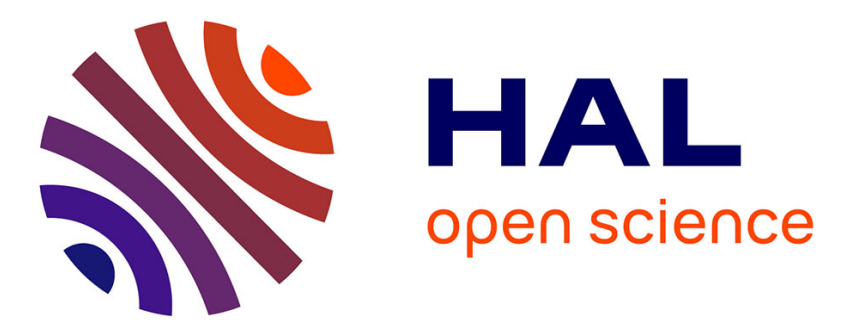

\title{
Thermal transfer in mixtures of ethylene glicol with carbon coated iron nanoparticles under the influence of a uniform magnetic field
}

Caridad Vales-Pinzon, Ruben Medina-Esquivel, J Ordonez-Miranda, Juan Jose Alvarado-Gil

\section{To cite this version:}

Caridad Vales-Pinzon, Ruben Medina-Esquivel, J Ordonez-Miranda, Juan Jose Alvarado-Gil. Thermal transfer in mixtures of ethylene glicol with carbon coated iron nanoparticles under the influence of a uniform magnetic field. Journal of Alloys and Compounds, 2014, 643 (Supplément 1), pp.S71-S74. 10.1016/j.jallcom.2014.12.057 . hal-01357550

\author{
HAL Id: hal-01357550 \\ https://hal.science/hal-01357550
}

Submitted on 11 Sep 2016

HAL is a multi-disciplinary open access archive for the deposit and dissemination of scientific research documents, whether they are published or not. The documents may come from teaching and research institutions in France or abroad, or from public or private research centers.
L'archive ouverte pluridisciplinaire HAL, est destinée au dépôt et à la diffusion de documents scientifiques de niveau recherche, publiés ou non, émanant des établissements d'enseignement et de recherche français ou étrangers, des laboratoires publics ou privés. 


\title{
Thermal transfer in mixtures of ethylene glicol with carbon coated iron nanoparticles
}

\section{under the influence of a uniform magnetic field}

\author{
C. Vales-Pinzon ${ }^{1, *}$, R.A. Medina-Esquivel ${ }^{2}$, J. Ordonez-Miranda ${ }^{3}$, J.J. Alvarado-Gil ${ }^{1}$ \\ ${ }^{1}$ Applied Physics Department, CINVESTAV-Unidad Mérida, Carretera Antigua a Progreso Km 6, Cordemex, Mérida, Yucatán 97310, Mexico \\ ${ }_{2}^{2}$ Facultad de Ingeniería-UADY, Av. Industrias no Contaminantes por Periférico Norte, Apdo. Postal 150 Cordemex, Mérida, Yucatán 97310, Mexico \\ ${ }_{3}^{3}$ Laboratoire d'Énergétique Moléculaire et Macroscopique, Combustion, UPR CNRS 288, Centrale Paris, Grande Voie des Vignes, 92295 Châtenay Malabry, France
}

\section{A b s t r a c t}

In this work, the study of the heat transfer enhancement induced by aligning iron nanoparticles in an ethylenglicol fluid matrix is presented. In particular the effect of the interface is studied by analyzing the cases in which the nanoparticles are coated with carbon and comparing with uncoated ones. Results indicate that the coating acts as a thermal barrier making thermal conductivity to decrease. Moreover, the magnetic field creates aligned columns that enhance the heat transfer. Effective models are used to deter-mine the role of the coating as well as of the aligning of the nanoparticles.

\section{Introduction}

Thermal conductivity of randomly suspended spheres in a matrix material has been the focus of a large number of investigations [1]. The thermal conductivity of fluids, developed for heat exchangers, plays a vital role in the development of energy-efficient heat transfer equipment. The thermal conductivity of most of the fluids is generally small. Applications in diverse fields, especially in heat exchangers demand the development of fluids with enhanced thermal conductivity [2,3]. One of the simplest configurations, helpful in reaching this objective, is to insert metallic particles in the fluids. If those metallic particles have a high magnetic moment they can get aligned with an external magnetic field. Heat transfer is expected to grow preferentially along the direction of the magnetic field, allowing in some way to manipulate the direction in which the heat is transferred. However, heat transfer from particle to particle could be affected by the thermal interface resistance. According to Maxwell model, the effective thermal conductivity of materials that contain spherical particles can be determined by simply considering the volume fraction concentration and the thermal conductivity of matrix and suspended particles. Nevertheless, effects of microstructural features of these composites as well as the interface thermal resistance play a determinant role on their macroscopic properties. In recent years, a lot of effective conductivity models have been developed in which particle interactions, size and form have been taken into account. However the experimental evaluation of the effects of the thermal interface resistance, among the nanoparticles and the fluid, are hard to evaluate. In this paper it is shown that, preparing ferrofluids with coated nanoparticles and comparing with ferrofluids with non-coated nanoparticles can be useful in the understanding of the role of thermal interface resistance in heat transfer.

\section{Materials and methods}

\subsection{Sample preparation}

Iron Nanoparticles (Fe, 99.5\%, passivated) with an average particle size of $25 \mathrm{~nm}$ and Iron nanoparticles carbon coated (Fe, 99.6\%) with an average particle size of 25 $\mathrm{nm}$ and an average carbon thickness of $2 \mathrm{~nm}$ (Nanostructured and Amorphous Materials) and ethylene glycol as carrier fluid were used to develop the studied samples. TEM images of the nanoparticles, supplied by the manufacturer, are shown in Fig. 1. The samples were prepared by one-step technique, at different nanoparticles volume fraction concentrations of $0 \%, 0.25 \%, 0.5 \%, 1 \%, 2.5 \%, 5 \%$ and $10 \%$. The nanoparticles were added to the ethylene glycol and then this mixture was sonicated with an ultrasonic processor working at $20 \mathrm{kHz}$.

\subsection{Experimental set up}

The thermal diffusivity was measured as a function of nanoparticles content. The samples were inserted inside a pair of Helmholtz coils and measurements were made when a magnetic field is turned off and compared with measurements with the magnetic field at 300G in the direction of the heat transport, using the Thermal Wave Resonator Cavity (TWRC). This is a very useful technique because it provides simple, versatile and accurate measurements of thermal properties of fluids [4]. We verify that the pyroelectric sensor is insensitive to the applied magnetic field by measuring the thermal diffusivity of non-magnetic fluids and we found no-difference when the field is on and off. The experimental setup for the measurement of the thermal diffusivity is shown in Fig. 2.

After determining the thermal diffusivity $\alpha$ of the samples, the effective thermal conductivity $k$ was obtained using the relation

$k=\alpha\left(\rho_{m} C_{m}(1-\phi)+\rho_{p} C_{p} \phi\right)$ 
where $C_{m}$ and $C_{p}$ are the specific heat capacity and $\rho_{m}$ and $\rho_{p}$ are the density of the matrix and nanoparticles respectively and the values of these parameters are shown in Table 1.

\section{Results and discussion}

Fig. 3 illustrates the images of the samples at different nanoparticle concentration. Fig. 3a and care samples of ethylene glycol at 0.25\% and $1 \%$ of carbon coated Fe nanoparticles, respectively. It is easy to observe agglomeration of the particles in the sample, due to the hydrophobic behavior of the carbon coating [1]. However, for samples at the same concentration of non-coated Fe nanoparticles, the samples did not show such degree of agglomeration (Fig. $3 \mathrm{~b}$ and d).

Thermal conductivity of the analyzed samples as a function of the nanoparticle concentration is shown in Fig. 4, for the cases in which the particles have a random spatial distribution and a chainlike structure in presence of the applied magnetic field (300G). It can be seen that the increment of the volume concentra-tion of nanoparticles causes an increasing in thermal conductivity. This could be expected due the fact that the thermal conductivity of the nanoparticles is larger than the thermal conductivity of the matrix [8]. At zero magnetic field and for a concentration of $5 \%$ of $\mathrm{Fe}$ nanoparticles, the increment in thermal conductivity is around 29\%, whereas for samples of carbon coated nanoparticles, the coating reduces the increment of the thermal conductivity, such that for the same concentration of nanoparticles, the incre-ment observed is only $9.3 \%$.

When the magnetic field has a direction along the axis of the thermal wave cavity cylinder, an enhancement of the thermal diffusivity and conductivity is observed. This may be associated to the fact that the particles form chain-like structures aligned in direction of the magnetic field, which facilitates the heat conduction along the direction parallel to the magnetic field [9]. The increment of the thermal conductivity due to the magnetic field for samples depend of the volumen concentration. The enhancement for the samples of Fe nanoparticles is about $4.6 \%$ for the higher concentration, while for carbon coated Fe nanoparticles at same concentration, the change is around 1.5\%, compared to the measurements without the magnetic field.

Two models are used to interpret the experimental data for the normalized thermal conductivity of a two-phase system. The models consider the effects of the microstructures of the samples, maximum packing fraction of the dispersed phase, interfacial thermal resistance and the ratio of the thermal conductivity of the nanoparticles with respect to the thermal conductivity of the matrix, among others.

\subsection{Model for particulate composites with oriented particles}

Let us consider a composite made up of spherical particles forming chain-like structures with a random orientation, as shown in Fig. 1. The overall thermal conductivity $k$ of this composite can therefore be modeled in two steps: First, we consider each chainlike structure with a straight shape as a single cylindrical particle and calculate its effective thermal conductivity $k_{p e}$. The random distribution of these effective particles is then used to determine $k$, in the second step. Given that the spherical particles are touching each other within the chain-like structures, $k_{p e}$ is given by the well-known series rule, as follows

$\frac{2 a}{k_{p e}}=\frac{2 a}{k_{p}}+\frac{1}{R}$,

where $a$ and $k p$ are the radius and thermal conductivity of the particles and $R$ is the particle-particle interface thermal resistance.

For a low volume fraction $f$ of particles $(f<15 \%)$, as is the case of interest in the present work, the thermal conductivity $k$ of the composite with a random distribution of cylindrical particles is then given by $[10,11]$

$$
\frac{k}{k_{m}}=\frac{3+(A+B) f}{3-A f}
$$

where $k_{m}$ is the thermal conductivity of the matrix and

$A=2 \frac{1-\lambda}{1+\lambda}, \quad B=\frac{k_{p e}}{k_{m}}-1, \quad \lambda=\frac{k_{m}}{k_{p e}}+\frac{a_{K}}{a}$

being $a_{K}=\rho k_{m}$ the so-called Kapitza radius $(12.8 \mathrm{~nm}$ for Fe and $28.5 \mathrm{~nm}$ for $\mathrm{C}$ ) and $\rho$ the interfacial thermal resistance among the matrix and particles.

\subsubsection{Coating effect}

If the spherical particles are coated with a coating of thickness $\delta$, Eqs. (2)-(4) still hold provided that the thermal conductivity $k_{p}$ of the uncoated particle is replaced by the effective thermal conductivity $k_{c p}$ of the coated spheres $\left(k_{p} \rightarrow k_{c p}\right)$, which can be written as $[10,11]$

$\frac{k_{c p}}{k_{c}}=1+\frac{3 v\left(k_{p}-k_{c}\right)}{3 k_{c}+(1-v)\left(k_{p}-k_{m}\right)}$

where $v=(a /(a+\delta))^{3}$ and $k_{c}$ is the thermal conductivity of the coating. Given that the thickness $\delta$ might be much smaller than the mean free path $l$ of the energy carriers within the coating, the thermal conductivity $k_{c}$ should take into account the boundary scattering of these carriers (size effects), as follows

$$
k_{c}=\frac{K_{c}}{1+l /(\delta+a / 2)},
$$

where $K_{c}$ is the bulk thermal conductivity of the coating material and it has been assumed that $\delta \ll a$ as is usually the case of practical interest.
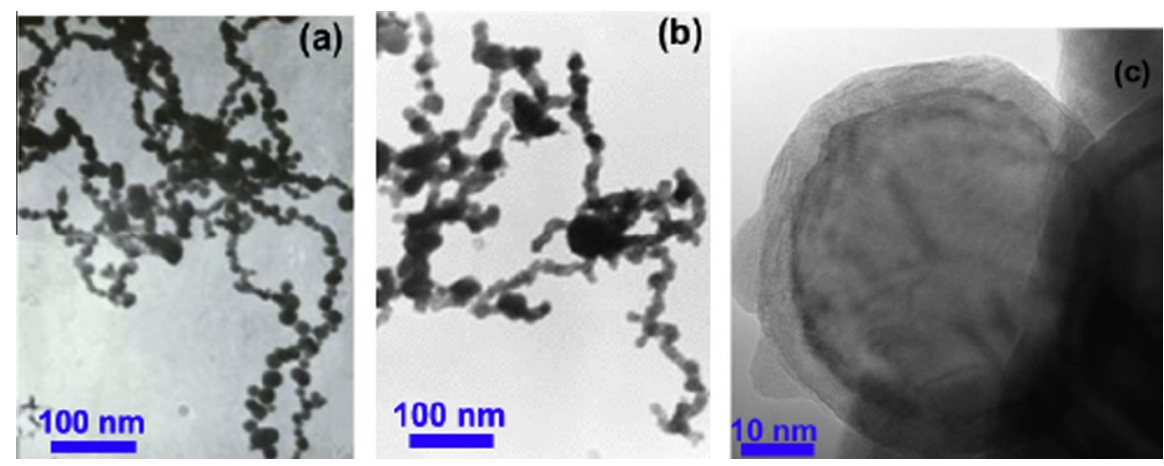

Fig. 1. TEM images of (a) Fe nanoparticles and (b and c) carbon coated Fe nanoparticles. 


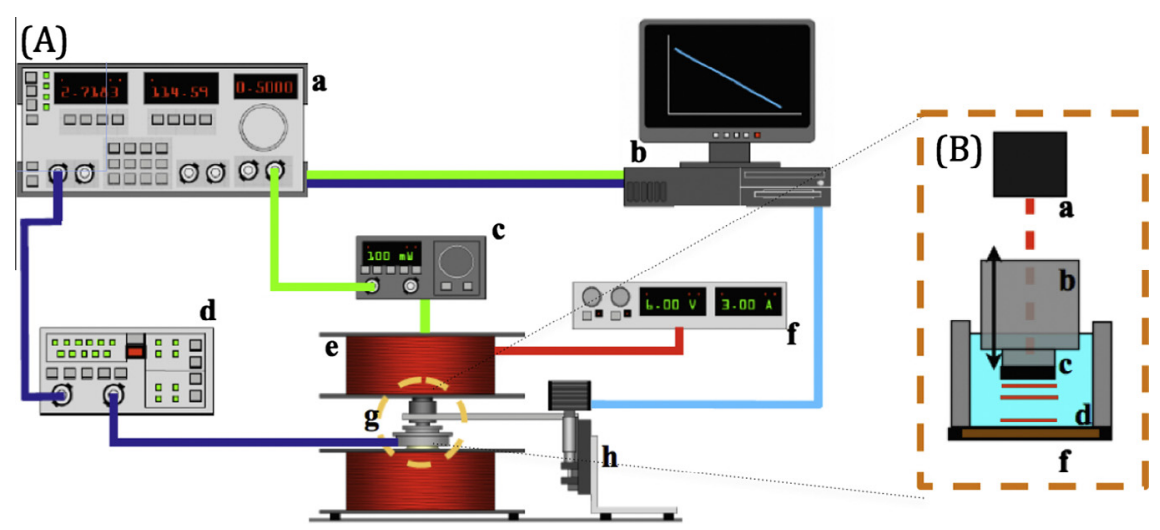

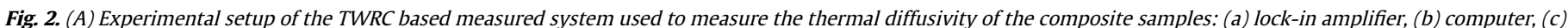

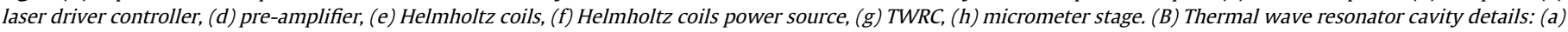
laser diode, (b) cylindrical thermal-wave emitter head containing the silicon wafer (c). (d) Sample container. (f) Pyroelectric sensor.

Table 1

Material properties used in the calculations[5-7].

\begin{tabular}{llll}
\hline Material & $\begin{array}{l}\text { Bulk thermal } \\
\text { conductivity }\left(\mathrm{Wm}^{-1} \mathrm{~K}^{-1}\right)\end{array}$ & $\begin{array}{l}\text { Density } \\
\left(\mathrm{kg} / \mathrm{m}^{-3}\right)\end{array}$ & $\begin{array}{l}\text { Specific heat capacity } \\
\left(\mathrm{Jkg}^{-1} \mathrm{~K}^{-1}\right)\end{array}$ \\
\hline $\mathrm{Fe}$ & 80.4 & 7870 & 450 \\
$\mathrm{C}$ & 1000 & 2000 & 710 \\
Ethylene glycol & 0.24 & 1113 & 2360 \\
\hline
\end{tabular}
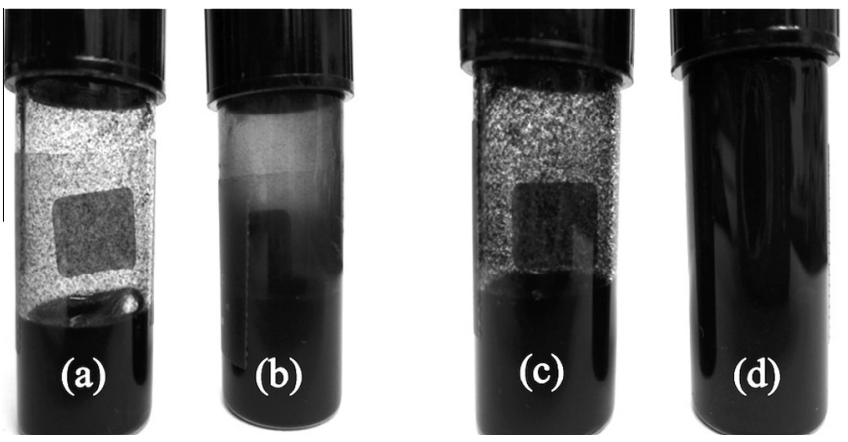

Fig. 3. Samples of ethylene glycol at $0.25 \%$ of (a) carbon coated Fe nanoparticles and (b) non-coated Fe nanoparticles and at $1 \%$ of (c) carbon coated Fe nanoparticles and (d) non-coated Fe nanoparticles.

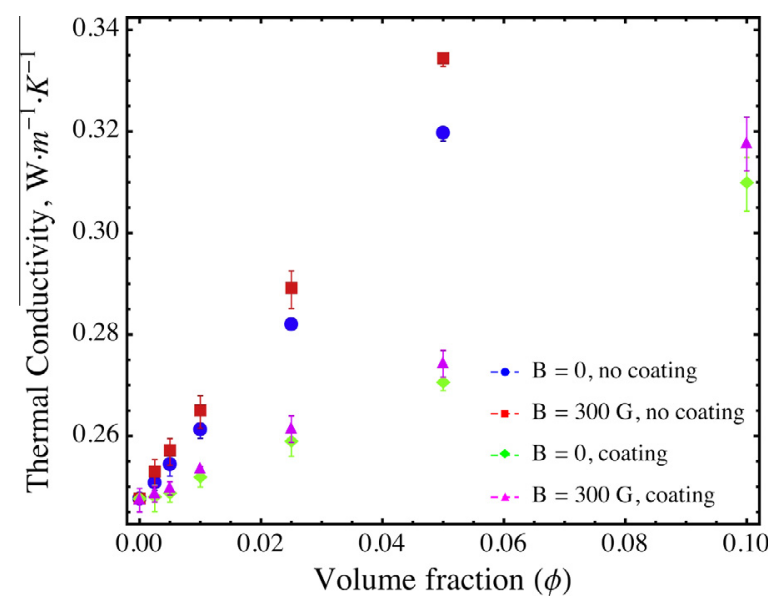

Fig. 4. Thermal conductivity of the samples in function of volume concentration of nanoparticles.

\subsubsection{Magnetic field effect}

If the spherical particles are based on iron, in presence of a magnetic field, the chain-like structures tend to align along the direction of the applied magnetic field $B$, which in turn will increase the effective thermal conductivity of each structure and of the composite, along that direction. In general, it is hard to know the degree of alignment of the chain-like structures for a given magnetic field; therefore the effective thermal conductivity of one of those structures can be written as $\gamma k_{p e}$, where $\gamma>1$ is a fitting parameter. The value of this parameter can be determined by fitting a theoretical model to the experimental data for the composite thermal conductivity in the direction perpendicular to $B$. This calculated value should be able to predict the composite thermal conductivity parallel to $B$, through Eqs. (2) and (3) under the replacement $\left(k_{p e} \rightarrow \gamma k_{p e}\right)$. This methodology is an extension of the one proposed by Reinecke et al. [2]

\subsection{A crowding factor model}

Another theoretical model, proposed by Ordonez-Miranda et al. [12], which considers the effects of both interfacial thermal resistance and is based on a crowding factor and is applicable for both low and high concentration of particles, up to their maximum packing fraction, can be used to analyze the normalized thermal conductivity. For this model, the thermal conductivity can be considered as follow

$\frac{k}{k_{m}}=\exp \left(\frac{3 A^{*} f}{1-A^{*} \Psi f}\right)$

where

$A^{*}=\frac{1-\left(k_{m} / k_{p e}+a_{K} / a\right)}{1+2\left(k_{m} / k_{p e}+a_{K} / a\right)}, \quad \Psi=1+\frac{\left(1-f_{0}\right)}{f_{0}^{2}} f$

where $f_{0}$ is the maximum packing fraction of the particles whose values are reported in literature for different types of particles and packing [13]. For spherical particles with a random distribution, $f_{0}=63.7 \%$. In similar way to the previous model, the factor of the Crowding model takes into account the carbon coating of the nanoparticles of Fe replacing $\left(k_{p} \rightarrow k_{c p}\right)$ according to Eq. (5). While for alignment of the structures due to the presence of a magnetic field, a fitting parameter $\gamma$ is considered, changing the thermal conductivity of the nanostructures, in such a way $\left(k_{p e} \rightarrow \gamma k_{p e}\right)$ is used.

Fig. 5 shows the normalized thermal conductivity of the samples in function of the nanoparticles concentrations and the predictions of the proposed models. The model that considers chain-like structures in random orientation and structures oriented in the 


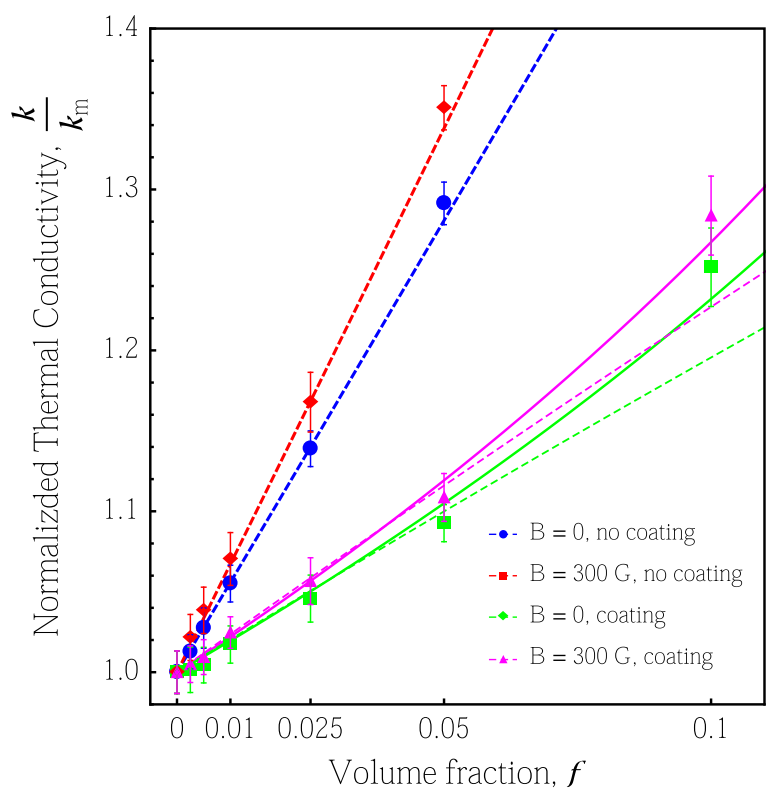

Fig. 5. Normalized thermal conductivity as a function of the volume fraction of particles and the comparison of the predictions of Eq. (3) with experimental data (dashed lines) and crowding factor model (solid lines).

direction of the magnetic field shows a good agreement with the experimental data for samples of Fe nanoparticles and carbon coated Fe nanoparticles at volume concentrations up to 5\%. However for carbon coated Fe nanoparticles samples at higher concentrations (above 5\%), the model presented in 3.1 do not provide a good fitting of the experimental data. In this case, the Crowding Factor model shows a good agreement with the experimental results due to the consideration of the particles interactions. In contrast the Crowding factor model does not provide a good fitting to the non-coated iron nanoparticles case. This could be due to the non-aglomeration behavior of these samples at the analyzed volume fractions studied.

\section{Conclusion}

Ethylene glycol based samples containing Fe nanoparticles and carbon coated Fe nanoparticles were prepared and their effective thermal conductivities were studied. The thermal conductivity of samples increases due to the increment of the volume concentration of both type of nanoparticles. This increment is improved when the sample is under a constant and uniform magnetic field so that the thermal conductivity has an enhancement of 11\%respect to the matrix by dispersing only 5\% (volume fraction) of carbon coated Fe nanoparticles in ethylene glycol. Whereas an increase of 35\% in thermal conductivity are achieved with only a load of 5\% of $\mathrm{Fe}$ nanoparticles. For mixtures of Fe particles and car-bon coated Fe nanoparticles, chain-like model shows a good agree-ment with the experimental results at concentrations up to 5\%. For samples with coated particles, the Crowding Factor model, that considers agglomeration effects, provides a good fitting for a broad range volume concentrations.

\section{Acknowledgements}

This work was supported by Conacyt-CeMIESol-COSOLpi and Cinvestav Grants. C. Vales-Pinzón acknowledges CONACYT for a PhD fellowship. We are grateful with M.Sc. J. Bante-Guerra for electronic and technical support.

\section{References}

[1] H. Zhang, Q. Wu, J. Lin, J. Chen, Z. Xu, Thermal conductivity of polyethylene nanofluids containing carbon coated metal nanoparticles, J. Appl. Phys. 108 (2010) 124304

[2] B.N. Reinecke, J.W. Shan, K.K. Suabedissen, A.S. Cherkasova, On the anisotropic thermal conductivity of magnetorheological suspensions, J. Appl. Phys. 104 (2008) 023507.

[3] X.W. Wang, X.F. Xu, S.U.S. Choi, Thermal conductivity of nanoparticle-fluid mixture, J. Thermophys. Heat Tansfer 13 (4) (1999).

[4] J. Shen, A. Mandelis, Thermal wave resonator cavity, Rev. Sci. Instrum. 66 (10) (1995).

[5] C. Kittel, Introduction to Solid State Physics, Wiley, 2005.

[6] E.T. Swartz, R.O. Pohl, Thermal boundary resistance, Rev. Sci. Instrum. 61 (3) (1989) 605.

[7] G.D. Mahan, Kapitza thermal resistance between a metal and nonmetal, Phys. Rev. B 79 (2009) 075408.

[8] R. Pal, New models for thermal conductivity of particulate composites, J. Reinf. Plast. Compos. 26 (7) (2007) 643-651.

[9] Qiang Li, Yimin Xuan, Jian Wang, Experimental investigations on transport properties of magnetic fluids, Exp. Thermal Fluid Sci. 30 (2005) $109-116$.

[10] J. Ordonez-Miranda, R.G. Yang, J.J. Alvarado-Gil, A model for the effective thermal conductivity of metal-nonmetal particulate composites, J. Appl. Phys. 111 (2012) 0443319.

[11] C.W. Nan, R. Birringer, D.R. Clarke, H. Gleiter, Effective thermal conductivity of particulate composites with interfacial thermal resistance, J. Appl. Phys. 81 (1997) 6692.

[12] J. Ordonez-Miranda, R.G. Yang, J.J. Alvarado-Gil, A crowding factor model for the thermal conductivity of particulate composites at non-dilute limit, J. Appl. Phys. 114 (2013) 064306.

[13] L.E. Nielsen, The thermal and electrical conductivity of two phase systems, Ind. Eng. Chem. Fundam. 13 (1) (1974) 17-20. 